\title{
Cytogenetical Studies on the Genus Sesbania Scop. I. Karyotype
}

\author{
R. S. Parihar and S. N. Zadoo \\ Indian Grassland and Fodder Research Institute, Jhansi, India
}

Accepted April 30, 1986

The genus Sesbania Scop. (Leguminosae) encompasses 50 species distributed in the tropics and sub-tropics. The first cytological studies on genus Sesbania were carried out by Kawakami (1930) followed by Senn (1938) who reported $2 \mathrm{n}=32$ in $S$. aculeata Poir. (=S. bispinosa Jacq.). In this species, chromosome number, $2 \mathrm{n}=12$ was reported by Rao (1946), Turner (1955), Miege (1960), Bir and Sidhu (1967), Baquar and Akhtar (1968) and Sareen and Trehan (1979). Whereas Jacob (1941), Haque (1946), Sampath (1947), Turner (1955), Datta and Sen (1960) and Dana and Datta (1961) found $2 n=24$. Aneuploid types with $2 n=13$ and 14 were reported in this species by Bir et al. (1975) and Bir and Sidhu (1981) besides the normal cytotypes.

In S. sesban (L.) Merr. (=S. aegyptiaca Poir.) $2 \mathrm{n}=12$ was reported by many workers (Jacob 1941, Rao 1946, Haque 1946, Sampath 1947, Turner 1955, Pawar and Kulkarni 1955, Berger et al. 1958, Baquar et al. 1965, Bir and Sidhu 1967, Datta and Neogi 1970, Al Mayah and Al Shehbaz 1977 and Sareen and Trehan 1979). Baquar and Akhtar (1968) found variation in haploid chromosome number $n=6$ and 7 in var. 'bicolor', $n=6,7$ and 8 in var. 'picta' and var. 'sesban' of this species. Such type of variation was also reported by Bir et al. (1975) who found $\mathrm{n}=6,7$ and 12 in var. 'bicolor', $\mathrm{n}=6,7,8$ and 14 in var. 'picta' and $\mathrm{n}=7$ and 8 in var. 'sesban'. Atchinson (1949) and Pawar and Kulkarni (1955) reported $2 \mathrm{n}=12$ in S. macrocarpa Muhl.

In $S$. grandiflora Pers. $\mathrm{n}=7$ was reported by Krishnaswamy and Rangaswamy Ayyangar (1935) and Senn (1938), whereas later reports of Jacob (1941), Rao (1946), Haque (1946), Sampath (1947), Tjio (1948) and Baquar and Akhtar (1968) established the chromosome number of this species to be $2 n=24$.

In the present study karyotypes have been analysed in one variety each of $S$. sesban (L.) Merr. (=S. aegyptiaca Pers.), S. aculeata Pers. (=S. bispinosa Jacq.), S. grandiflora Poir. and two varieties representing two cytotypes of $S$. macrocarpa Muhl.

\section{Material and methods}

The seeds of five taxa representing the four species of the genus Sesbania, one each of $S$. aculeata, $S$. sesban, $S$. grandiflora and two cytotypes of $S$. macrocarpa have been taken for karyotypic analysis.

For mitotic studies seeds were germinated in petridishes and young healthy root tips were washed and pre-treated for $3 \frac{1}{2}$ hours in a saturated aqueous solution of para-dichlorobenzene at room temperature, washed in water for about five minutes and fixed in $1: 3$ acetic-alcohol for 3 hours. The toot tips were again washed in water and hydrolysed for 12 minutes in $\mathrm{N} \mathrm{HCl}$ at $60^{\circ} \mathrm{C}$ and finally stained in leuco-basic fuchsin for about half an hour. Stained tips were squashed in a drop of 1 per cent acetocarmine.

Karyotypes from root meristem were analysed from a minimum five cells for arm ratio and other morphological details. Photo-idiograms were prepared from photomicrographs

1 Part of the Ph. D. Thesis submitted by R. S. Parihar to the Bundelkhand University, Jhansi, India. 
by cutting out individual chromosomes, arranging them in descending order of their length and matching on the basis of morphology. Battaglia's (1955) classification of metacentric (V), submetacentric (L), sub-telocentric (J) and telocentric (I) based on arm ratio $1: 1,>1: 1$ to $<$ $1: 3,>1: 3$ to $<0: 1$, and $0: 1$ respectively was used to determine the exact position of centromere. Stebbins' (1958) method was used for assessing the degree of asymmetry.

\section{Observations}

Five taxa, i.e. Sesbania sesban var. 'bicolor', S. aculeata 'IL 2264', S. macrocarpa 'IL 1535', $S$. macrocarpa 'IL 89' and $S$. grandiflora 'IL 211 ' were studied for their mitotic complements. Out of these, three taxa were diploid with $2 n=12$ and two taxa were tetraploid with $2 n=24$ chromosomes in their mitotic complement.

\section{Diploids}

Twelve chromosomes in the somatic complement could be grouped in 6 sets of two chromosomes each. There were no abrupt changes in the size of different sets, and chromosomes formed a gradually graded series from longest to shortest set.

\section{S. aculeata 'IL 2264'}

The longest chromosome pair of the complement of this species is 2.2 times as long as the shortest pair. First and fifth pairs were with submedian centromeres, second and third pairs had subterminal centromeres while fourth and sixth sets of chromosomes were with median centromeres. Nucleolar organisers were found to be in the form of satellites located on short arms of three (second, third and fifth) pairs of chromosomes. The karyotypic formula for the taxa worked out to be $4 \mathrm{~V}+4 \mathrm{~L}+4 \mathrm{~J}$ (Fig. 1, Table 1).

$S$. sesban var. 'bicolor'

The longest chromosome pair of the complement in this taxon was about two times longer than the shortest pair. The first two homologous pairs in this species were with submedian centromeres. The third, fourth and sixth pairs have centromeres in exactly median position. Nucleolar organisers in the form of satellites were located on short arm of the fifth homologous pair of chromosomes having centromere in submedian position. The karyotypic formula worked out to be $6 \mathrm{~V}+6 \mathrm{~L}$ (Fig. 2, Table 1).

S. macrocarpa 'IL 1535'

The longest chromosome pair of the complement in this species was two times longer than the shortest chromosome. The first three homologous pairs were with submedian centromeres and last three homologous pairs were with median centromeres. Nucleolar organisers were in the form of satellites located on fifth homologous pair of chromosomes. The karyotypic formula worked out to be $6 \mathrm{~V}+6 \mathrm{~L}$ (Fig. 3, Table 1).

\section{Tetraploids}

Sesbania macrocarpa 'IL 89' and S. grandiflora 'IL 211' studied presently are tetraploids with $2 \mathrm{n}=24$ chromosomes in the somatic complement. Like diploids the chromosomes form a graded series from longest to shortest chromosomes of the complement and there are no abrupt changes in chromosomal size. The chromosomes could be resolved into six groups of four chromosomes each, however, slight differences in exact centromere position were noted in different pairs in a group of four chromosomes. As such for the sake of discription they have been taken as individual pairs I to XII.

S. macrocarpa 'IL 89'

All the chromosome sets were homomorphic. The largest pair of chromosomes was about three times as large as the smallest pair of chromosomes. First six pairs of chromosomes were 


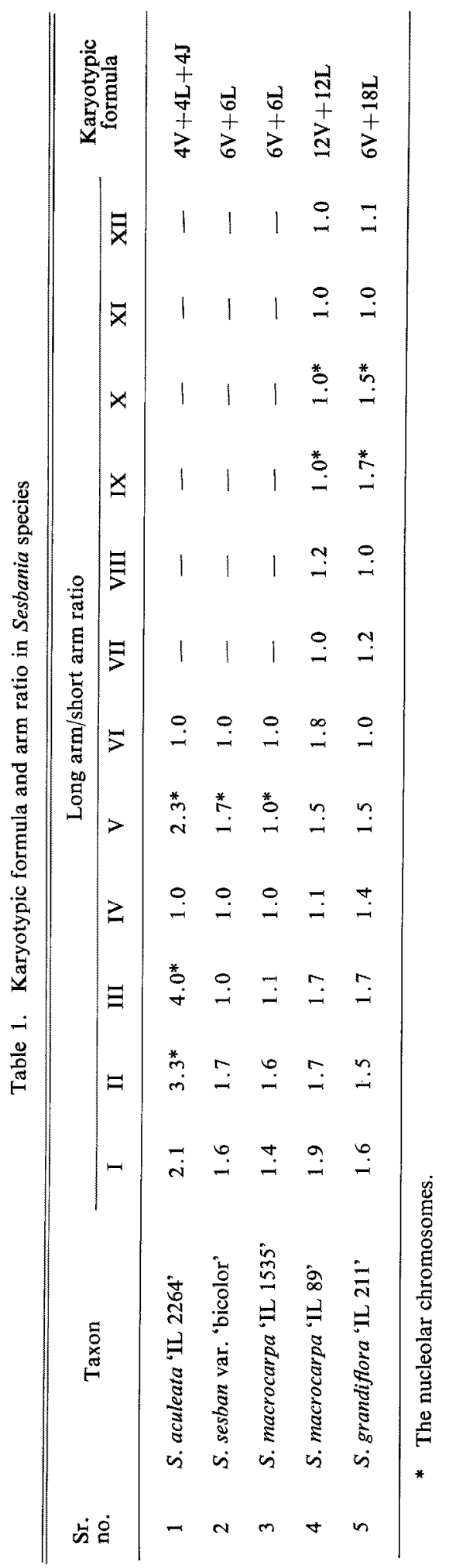


with submedian centromeres, while the last six pairs had centromeres in median position. Nucleolar organizers in the form of satellites were observed on ninth and tenth homologous pairs of chromosomes. The karyotypic formula worked out to be $12 \mathrm{~V}+12 \mathrm{~L}$ (Fig. 4, Table 1).

S. grandiflora 'IL 211'

All the sets were homomorphic. The longest pair of chromosome in this species was about two times longer than the shortest chromosome. Sixth, eighth and eleventh pairs were metacentric having centromere exactly in median position. Rest all the nine pairs of chromosome were with submedian centromeres. Nucleolar organisers were observed in two pairs of chromosomes in the form of satellites located on the short arm of the ninth and tenth homologous pairs. Both the pairs of nucleolar chromosomes were with submedian centromere. The karyotypic formula worked out to be $6 \mathrm{~V}+18 \mathrm{~L}$ (Fig. 5, Table 1).

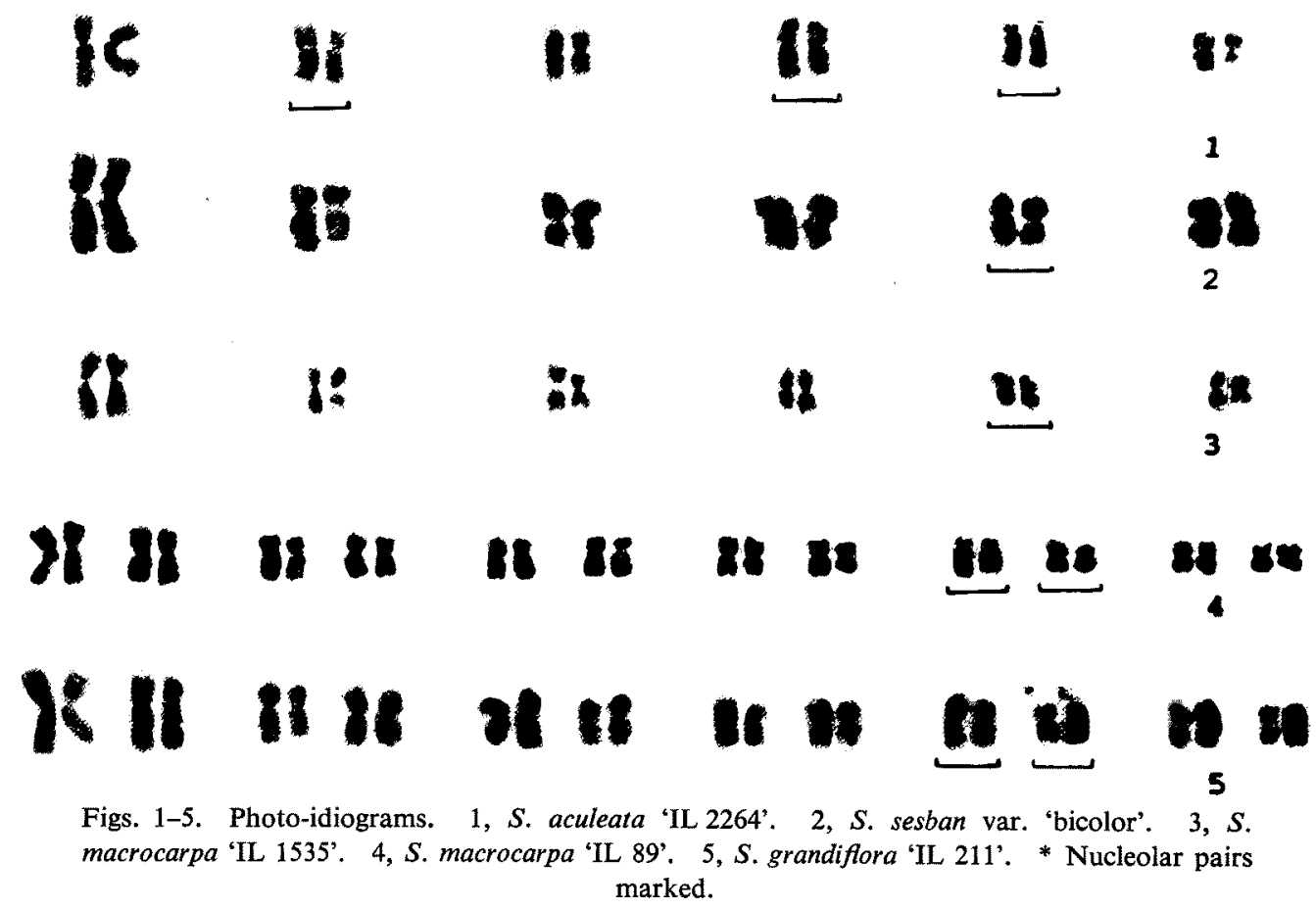

\section{Discussion}

Karyotypic analysis of representative varieties of the species and diploid and tetraploid cytotypes in Sesbania reveals that the genus has essentially a symmetrical karyotype. The magnitude of karyotype asymmetry in various taxa has been determined by parameters of relative size of chromosomes and position of centromere following the system proposed by Stebbins (1958). The complement under study is predominantly composed of median and submedian chromosomes, which are essentially primitive karyotypes and fall in $1 \mathrm{~A}$ or $2 \mathrm{~A}$ types of Stebbins' classification (1958). It may be pertinent to add here that perennial species represented by $S$. sesban and $S$. grandiflora are characterised by chromosomes with only median and submedian chromosomes, while herbaceous annual species like $S$. aculeata have 2 pairs of subterminal chromosomes in their complement. Herbaceous diploid and tetraploid S. macrocarpa, however, have only median/submedian chromosomes in the complement. The complement of $6 \mathrm{~V}+6 \mathrm{~L}$ reported here is in line to that reported by Sareen and Trehan (1979) for S. sesban var. 
'bicolor'. The present results are not in confirmity with those of above authors in case of $S$. bispinosa $(=S$. aculeata) who reported $6 \mathrm{~V}+6 \mathrm{~L}$, while our finding shows a complement of $4 \mathrm{~V}+$ $4 \mathrm{~L}+4 \mathrm{~J}$. Bir and Sidhu (1981) worked out the karyotypes of three cytotypes of $S$. bispinosa with $2 \mathrm{n}=12,13$ and 14 chromosomes respectively. They found $4 \mathrm{~V}+8 \mathrm{~L}$ in cytotype with $2 \mathrm{n}=$ 12 chromosomes. An analysis of cytotypes with 13 and 14 chromosomes revealed that the extra chromosome was morphologically similar to metacentric chromosome of 4 th homologous pair of normal cytotype with an $=12$. On the other hand in $2 n=14$ cytotype an additional pair of homologous chromosomes was present, giving a karyotypic formula of $5 \mathrm{~V}+8 \mathrm{~L}$ and $6 \mathrm{~V}+8 \mathrm{~L}$ respectively for cytotypes with $2 \mathrm{n}=13$ and 14 . The differences in the observations on the karyotype of S. bispinosa by Bir and Sidhu (1981) and the present authors may be due to the fact that the materials used in these two studies belong to different locations. The mitotic complements of diploid $S$. macrocarpa $(6 \mathrm{~V}+6 \mathrm{~L})$, tetraploid $S$ macrocarpa $(12 \mathrm{~V}+12 \mathrm{~L})$ and tetraploid $S$. grandiflora $(6 \mathrm{~V}+18 \mathrm{~L})$ have been recorded for the first time during the present studies.

No correlation was found between the number of nucleolar organising chromosomes and grade of polyploidy of different taxa. While six chromosomes (three homologous pairs) were found to be with nucleolar organisers in diploid species $S$. aculeata, their number was found to be only two in other diploid taxa studied presently, i.e. Sesbania sesban var. 'bicolor' and diploid cytotype of $S$. macrocarpa. In tetraploid taxa studied presently, viz., $S$. macrocarpa 'IL 89' and S. grandiflora 'IL 211', the number of nucleolar organising chromosomes was four. The nucleolar organisers were mostly in the form of satellites located on short arm of submedian chromosomes in case of $S$. sesban and $S$. grandiflora, median chrosomomes in S. macrocarpa, subterminal/submedian chromosomes in $S$. aculeata, while in tetraploid cytotype of $S$. macrocarpa nucleolar organisers are located on median chromosomes. In this respect the present studies are in line with Sareen and Trehan (1979) as far as number of nucleolar chromosomes is concerned in case of $S$. sesban, but differ in the sense that the said workers have found nucleolar organisers in the form of secondary constriction on longest pair of homologues. Jacob (1941) found as many as three pairs of nucleolar chromosomes in S. sesban var. "sesban' and $S$. sesban var. 'bicolor'. A single pair of nucleolar chromosomes has been found in $S$. sesban by Datta and Neogi (1970).

A single pair of nucleolar chromosomes has been reported by Dana and Datta (1961) and Sareen and Trehan (1979) in S. bispinosa, both of them reported secondary constriction on longest pair of chromosomes. While Dana and Datta (1961) reported this species as tetraploid $(2 \mathrm{n}=24)$, Sareen and Trehan (1979) worked on a diploid taxa. Our studies on $S$. aculeata $(=S$. bispinosa) revealed it to be a diploid with six nucleolar organising chromosomes.

It is apparent from above discussion that there is a lot of polymorphicity in karyotypes worked out by different workers, which warrants a detailed study of the material from different locations/populations.

\section{Summary}

Five taxa i.e. Sesbania aculeata 'IL 2264', S. sesban var. 'bicolor', S. macrocarpa 'IL 1535' and 'IL 89' and $S$. grandiflora 'IL 211' were studied for their mitotic complement. Out of these, first three taxa were diploid $2 n=2 x=12$ and the other two taxa were tetraploid with $2 n=4 x=$ 24 chromosomes in their mitotic complements.

The karyotypic formula was found to be $4 \mathrm{~V}+4 \mathrm{~L}+4 \mathrm{~J}$ in $S$. aculeata 'IL 2264 ', $6 \mathrm{~V}+6 \mathrm{~L}$ in $S$. sesban var. 'sesban' and S. macrocarpa 'IL 1535', 12V+12L in S. macrocarpa 'IL 89' and $6 \mathrm{~V}+18 \mathrm{~L}$ in $S$. grandiflora 'IL 211 '.

The nucleolar organisers were in the form of satellites located on short arm of fifth set of 
chromosomes. No correlation was found between the number of nucleolar organising chromosomes and grade of polyploidy of different taxa, while six chromosomes (three homologous pairs) were found to be with nucleolar organisers in diploid species $S$. aculeata. Their number was found to be only two in other diploid taxa studied presently i.e. $S$. sesban var. 'bicolor' and diploid cytotype $S$. macrocarpa 'IL 1535'. In tetraploid taxa i.e. S. macrocarpa 'IL 89' and $S$. grandiflora 'IL 211', the number of nucleolar organising chromosomes was four.

\section{Acknowledgement}

Thanks are due to Director, Indian Grassland and Fodder Research Institute, Jhansi for facilities and Dr. S. K. Gupta for going through the manuscript.

\section{References}

Al Mayah, A. R. A. and Al Shehbaz, I. A. 1977. Chromosome numbers for some Leguminosae from Iraq. Bot. Notiser 130: 437-440.

Atchison, E. 1949. Chromosome numbers and geographical relationship of miscellaneous Leguminosae. J. Elisha Mitchell Sci. Soc. 65: 118-130.

Baquar, S. R. and Akhtar, S. 1968. Cytological studies of the genus Sesbania from W. Pakistan. Cytologia 33: 427-438.

-, - and Husain, A. 1965. Meiotic chromosome numbers in some vascular plants of Indus Delta I. Botaniska Notiser 119: 24-32.

Battaglia, E., 1955. Chromosome morphology and terminology. Caryologia 8: 178-187.

Berger, C. A., Witkus, E. R. and McMohan, R. M. 1958. Cytotaxonomic studies in the Leguminosae. Bull. Torrey Bot. Club 85: 405-414.

Bir, S. S. and Sidhu, M. 1981. Cytological studies on weeds I. Sesbania bispinosa. Curr. Sci. 50: 281-282.

- and Sidhu, S. 1967. Cytological observations on the North Indian members of family Leguminosae. The Nucleus 10: 47-63.

-, - and Talwar, K. 1975. Cytological observations on genus Sesbania from the Punjab plains (North India). New Botanist 2: 101-108.

Dana, S. K. and Datta, R. M. 1961. Comparative cytological studies of the pollen tube in diploid and tetraploid species of Sesbania. New Phytol. 60: 285-311.

Datta, R. M. and Neogi, A. K. 1970. Chromosome numbers and karyotypes in the genera Crotalaria and Sesbania. Acta Agron. Acad. Sci. Hung. Tomies 19: 343-350.

- and Sen, S. K. 1960. Intraspecific hybridization between Sesbania aculeata Pers. (4n race) and S. speciosa Taub ex Engler ( $2 \mathrm{n}$ race) and causes of failure of viable seed formation. Züchter 30: 265-269.

Haque, A. 1946. Chromosome number in Sesbania. Curr. Sci. 15: 78-79.

Jacob, K. T. 1941. Cytological studies in the genus Sesbania. Bibliographia Genetica 13: 225-297.

Kawakami, J. 1930. Chromosome numbers in Leguminosae. Bot. Mag. Tokyo 44: 319-328.

Krishnaswamy, N. and Rangaswamy Ayyanger, G. N. 1935. Chromosome numbers in Sesbania grandiflora Pers. Curr. Sci. 3: 488.

Miege, J. 1960. Nombres chromosomique des plantes occidentales. Rev. Cyt. Biol. Vég. 21: 373-384.

Pawar, M. S. and Kulkarni, S. A. 1955. Chromosome number of Sesbania species. Curr. Sci. 24: 207-208.

Rao, Y.S. 1946. Chromosome numbers in Sesbania. Curr. Sci. 15: 78-79.

Sampath, S. 1947. Chromosome number in Sesbania spp. Curr. Sci. 16: 30-31.

Sareen, T. S. and Trehan, R. 1979. Karyotypes of some taxa in Sesbania Adanson. Acta. Bot. Indica 7: 178-180.

Senn, H. A. 1938. Chromosome number relationship in Leguminosae. Bibliographia Genetica 12: 175-336.

Stebbins, G. L. 1958. Longevity, habitat and release of genetic variability in higher plants. Cold Spr. Harb. Symp. Quant. Biol. 23: 365-378.

Tjio, J. H. 1948. The somatic chromosomes of some tropical plants. Hereditas 34: 135-146.

Turner, B. L. 1955. Chromosome numbers in the genus Sesbania (Leguminosae): Evidence for a conservative treatment. Rhodora 57: 213-218. 